\title{
Introduction
}

\section{Anne Fornerod}

\section{(2) OpenEdition}

\section{Journals}

Édition électronique

URL : https://journals.openedition.org/rdr/805

DOI : $10.4000 /$ rdr.805

ISSN : 2534-7462

Éditeur

Presses universitaires de Strasbourg

\section{Édition imprimée}

Date de publication : 3 mai 2017

Pagination : $9-14$

ISBN : 978-2-86820-973-3

ISSN : 2493-8637

\section{Référence électronique}

Anne Fornerod, "Introduction », Revue du droit des religions [En ligne], 3 | 2017, mis en ligne le 03 février 2020, consulté le 28 septembre 2021. URL : http://journals.openedition.org/rdr/805 ; DOI : https:// doi.org/10.4000/rdr.805

\section{(c) (i) (9)}

La revue du droit des religions est mise à disposition selon les termes de la Creative Commons Attribution - Pas d'Utilisation Commerciale 4.0 International - CC BY-NC 4.0. 


\section{INTRODUCTION}

\section{Anne FORNEROD}

Université de Strasbourg / CNRS, Droit, Religion, Entreprise et Société (DRES)

$\mathrm{D}$ ans la plupart des pays européens, le patrimoine culturel religieux, immobilier et mobilier, constitue une part significative de l'héritage historique et artistique ${ }^{1}$. Ces monuments familiers du paysage européen, tant rural qu'urbain, offrent un double visage, à la fois culturel et spirituel, qui renvoie à deux corpus de règles juridiques. En effet, le statut de biens utilisés à des fins de pratiques religieuses fait l'objet d'un encadrement légal spécifique, fondé sur la protection de la liberté de culte, qui relève des relations entre les pouvoirs publics et les groupes religieux. La destination première de ces édifices implique de les considérer à l'aune de la liberté de religion, dont l'effectivité repose, pour les groupes concernés, sur la possibilité de disposer de lieux spécifiquement consacrés aux célébrations religieuses. En outre, l'intérêt historique ou artistique reconnu à un monument entraîne l'applicabilité de la législation, souvent ancienne, sur les monuments historiques, ou, plus largement, de textes relatifs au patrimoine culturel dans son ensemble ${ }^{2}$. De là, il convient de considérer en parallèle un certain nombre d'éléments, qui, loin d'être statiques, connaissent des évolutions. D’une part, la notion de patrimoine culturel traduit un enrichissement du statut de

1. Ce qui relève du patrimoine immatériel et renvoie à des enjeux et problématiques bien spécifiques ne sera pas traité dans ce dossier.

2. L'appartenance au patrimoine culturel ne se limite en effet pas au statut de monument historique, mais peut résulter de l'application d'autres textes. Ainsi, le label français «Patrimoine du Xx siècle» a été créé en 1999 pour des biens qui ne sont pas obligatoirement protégés au titre des monuments historiques. 
monument historique : au-delà de l'objectif de protection et de conservation, il s'agit de transmettre un patrimoine à travers sa mise en valeur auprès du public, en permettant un accès aux biens qui le composent. Cette notion renvoie aussi à un phénomène d'appropriation par les individus et les groupes de biens, matériels et immatériels, en dehors des processus institutionnels de sélection patrimoniale. Il s'ensuit, s'agissant des édifices cultuels concernés, une double appréhension et une double fréquentation, d'ordre religieux au premier chef, mais faite aussi d'activités culturelles diverses. D'autre part, ces biens traversent une période de transition dont l'issue est encore incertaine, mais dont on sait qu'elle découle de l'évolution des pratiques au sein des traditions religieuses historiques, entraînant une utilisation moindre des édifices qui leur sont consacrés. Le scénario radical consistant à recourir à la destruction des édifices religieux devenus inutiles demeure bien rare au regard du nombre total de bâtiments, mais ce sont de telles situations qui donnent lieu à la plus forte médiatisation et renvoient à un horizon peu engageant.

Ce dossier vise à travers quelques contributions à interroger l'appréhension « classique » du patrimoine religieux, fondée essentiellement sur l'articulation variable entre les deux traits majeurs de leur régime juridique, le statut de lieu de culte d'une part, celui de biens culturels d'autre part. En France, l'affectation légale au culte des édifices religieux a des effets qui singularisent substantiellement la situation hexagonale. Aussi l'analyse mérite-t-elle d'être étendue à d'autres systèmes de relations religion-État, afin d'apprécier comment, d'un pays à l'autre, se joue cette transition majeure dans l'histoire de ces biens dont certains ont perdu leur usage premier. Si de nombreux pays partagent la même préoccupation consistant à préserver un patrimoine culturel, le statut des biens religieux est susceptible d'être influencé par celui des organisations religieuses défini dans un cadre national. L'enjeu principal est de mesurer les conséquences des évolutions des pratiques religieuses, dans un contexte de sécularisation mais également de pluralité. Aussi, s'intéresser au patrimoine culturel religieux aujourd'hui consiste pour l'essentiel à réfléchir à son avenir, interrogation qui peut se décliner et être abordée sous trois angles étroitement liés.

Il s'agit en premier lieu du financement, question qui se pose en des termes nouveaux à mesure que le patrimoine religieux se transforme. D'une part, la recherche de financements, qu'ils soient publics ou privés, demeure une préoccupation constante, qu'aiguise la raréfaction des ressources provenant des groupes religieux eux-mêmes. D'autre part, c'est la légitimité de la 
répartition entre subventions publiques et engagement financier des groupes religieux propriétaires ou usagers des édifices cultuels qui est interrogée. Sur ce point, si l'intervention d'un financement public est débattue, c'est moins dans son principe qu'en raison de son objet. La légitimité de la protection $\mathrm{du}$ patrimoine religieux peut suffire à justifier un soutien des collectivités publiques - surtout lorsqu'elles sont propriétaires - aux fins d'en assurer la conservation matérielle. Pour autant, la perspective que ce financement puisse bénéficier ne serait-ce qu'en partie à ce qui relève de l'exercice du culte est susceptible de soulever des réticences. La distinction et l'articulation entre ce qui ressort du financement de la conservation d'un bien au titre du patrimoine historique et culturel et ce qui équivaut à une aide à l'exercice du culte apparaît à première vue comme un questionnement étroitement associé à un régime de séparation entre l'État et les cultes (voir notamment Eva Löfgren, «Les défis de la conservation du patrimoine de l'Église de Suède : financement étatique et continuité de l'usage »), mais l'examen d'autres systèmes de relations État-religions vient relativiser cette analyse (voir Jean-François Husson, «Les pouvoirs publics et les édifices cultuels en Belgique »). Or, la recherche de cet équilibre peut être bousculée lorsque l'usage religieux disparaît. Peut-on, dans cette hypothèse, toujours alléguer que les mécanismes de soutien financier public, direct ou indirect, participent de la protection et de la mise en œuvre effective de la liberté de culte ? Si la baisse des pratiques religieuses qui concerne une proportion significative du patrimoine cultuel n'est pas un phénomène nouveau, l'investissement des personnes publiques ou privées qui en sont propriétaires, en période de crise financière, n'est sans doute plus aussi systématique que par le passé et une réutilisation à des fins autres que religieuses constitue une perspective davantage considérée. Car au-delà de l'objectif de parvenir à la conservation matérielle des biens culturels religieux, leur appartenance au patrimoine culturel entraîne une nouvelle signification, qui n'est plus exclusivement religieuse.

En effet, appréhender l'avenir du patrimoine cultuel suppose en deuxième lieu d'interroger le sens véhiculé par ces monuments dans un contexte de recomposition des appartenances religieuses et de leurs modes d'expression. La diminution des pratiques cultuelles "classiques » est susceptible de conférer aux biens qui en sont ou en ont été le support une nouvelle signification, qui mêle de façon variable «valeur patrimoniale» et «valeur religieuse ». L'approche contemporaine du patrimoine religieux est aussi déterminée par une diversification des pratiques religieuses, renvoyant à des enjeux différents. Ainsi, l'on observe de nouvelles religiosités qui se 
développent à l'écart des lieux de culte traditionnels. Le cas des sites naturels sacrés estoniens est révélateur de la complexité - et de la perplexité - qui naît de la gestion de biens éloignés des standards du patrimoine culturel religieux (Ringo Ringvee, "La protection du patrimoine religieux en Estonie : lieux de culte et sites naturels sacrés »). Ces formes moins conventionnelles du culte invitent à se tourner vers le caractère pluriel du patrimoine religieux dans son ensemble.

En réalité, les inquiétudes liées à la diminution, voire la disparition, des pratiques religieuses reposent sur celles qui, dans le cadre paroissial, incarnent l'exercice de la liberté de culte, protégée en droit. Or, l'on peut présager que ces pratiques «classiques » n'évolueront pas dans le sens d'un retour, même partiel, à l'état antérieur qui a présidé à l'adoption du régime juridique applicable. La disparition totale de l'usage religieux ne concerne qu'une part très minoritaire de l'ensemble du patrimoine, mais ouvre le dossier sensible des conversions d'édifices cultuels désacralisés et désaffectés. De manière générale, l'intérêt que représente un édifice du culte et qui justifie l'application de la législation sur les monuments historiques peut être établi selon des considérations étrangères à son usage. Dans ce cas de figure, la disparition, la diminution ou le changement de destination d'un édifice relevant de la législation sur les monuments historiques ne sont considérés qu'à raison d'une éventuelle répercussion sur l'intégrité physique et donc la conservation du bâtiment. L'hypothèse d'une singularité des monuments historiques religieux au sein de l'ensemble du patrimoine culturel repose essentiellement sur le maintien d'un usage originel dans des proportions inconnues d'autres «catégories » patrimoniales. L'usage originel disparu, où situer la spécificité du patrimoine religieux ? Dans quelle mesure observe-t-on un glissement vers le mode de gestion commun du patrimoine culturel ? L'identité religieuse devenue symbolique après désaffectation suffit-elle alors à influencer le droit applicable et à imprimer sa marque à l'appréhension de ce patrimoine?

Si juridiquement, la consécration du caractère religieux de ces biens repose traditionnellement et encore aujourd'hui sur un usage et des pratiques cultuels effectifs, la question se pose en troisième lieu de la forme juridique à donner à cette signification qui perdure au-delà de la disparition d'une fréquentation essentiellement rituelle et, plus largement, de l'adaptation du droit applicable aux mutations du patrimoine religieux, évoquées ici de façon non exhaustive. 
Sans refléter, loin de là, ni l'état général du patrimoine religieux, ni l'état d'esprit dans lequel il est globalement appréhendé, les « affaires » de destruction d'églises et de reconversions discutées n'en ont pas moins ouvert la voie à une réflexion sur l'avenir de milliers de bâtiments. Sur un plan juridique, l'évolution semble plus lente. Il est vrai que la problématique diffère à partir du moment où l'usage est maintenu et toujours consacré par le droit. Pour autant, un décalage s'instaure entre l'usage réel - ou plus exactement le non-usage - et le droit applicable, qui permet de protéger et prolonger juridiquement la dimension sacrée de l'édifice. Pendant de nombreuses années, l'enjeu a consisté, et consiste toujours, à concilier la double vocation de ces édifices et à organiser la cohabitation entre usages religieux et culturels. Il s'agissait là déjà d'une progression notable, qui dépassait la seule recherche d'un équilibre entre conservation du monument et utilisation religieuse (même s'il convient, de ce point de vue, de ne pas sous-estimer encore aujourd'hui la méconnaissance croissante du patrimoine religieux et de ce qu'il requiert, que ce soit sur le plan de la conservation ou de sa compréhension en tant que tel). Dans le contexte français, la situation particulièrement avantageuse des édifices du culte catholiques construits avant la séparation des Églises et de l'État du fait de leur appartenance au domaine public a longtemps été épargnée par un tel questionnement sur l'à-propos et la perpétuation d'un tel état du droit. Or, il n'est pas certain que, du développement des usages étrangers au culte aux destructions et reconversions d'églises, les transformations du patrimoine cultuel se reflètent dans le droit applicable - et tel qu'il est appliqué - lequel demeure protecteur de "l'intégrité » du patrimoine religieux (voir Stéphane Duroy, «Le patrimoine cultuel immobilier : un patrimoine en péril ?»). Les aménagements du dispositif légal hérité du processus de séparation reposent encore pour beaucoup sur une vision dualiste des édifices cultuels protégés au titre des monuments historiques, consistant à rechercher une articulation entre usage religieux et usages culturels (Pierre-Henri Prélot, «La valorisation patrimoniale des édifices religieux entre affectation cultuelle exclusive et contractualisation $»$ ).

Les questionnements - et surtout les réponses apportées jusqu'à présent - varient relativement peu d'un contexte national à un autre (voir Theodosios Tsivolas, "Sacredness as an underlying value of cultural heritage law in Europe »). Les exemples retenus ici montrent la prégnance d'une conception du patrimoine religieux où le sacré demeure longtemps présent, même après qu'il y ait été mis fin sur le plan juridique. En outre, l'on observe que le rôle incontournable des autorités religieuses continue 
d'imprimer une originalité certaine à la façon d'appréhender la valorisation d'ordre culturel des biens immobiliers et mobiliers concernés. Il y a donc plus d'un pas à franchir avant d'atteindre une réelle coincidence entre le régime juridique et la réalité d'un patrimoine religieux pluriel et en mutation. 